\title{
A general composite iterative method for strictly pseudocontractive mappings in Hilbert spaces
}

Jong Soo Jung*

\author{
"Correspondence: jungjs@dau.ac.kr; \\ jungjs@mail.donga.ac.kr \\ Department of Mathematics, \\ Dong-A University, Busan, 604-714, \\ Korea
}

\begin{abstract}
In this paper, we introduce a new general composite iterative method for finding a fixed point of a strictly pseudocontractive mapping in Hilbert spaces. We establish the strong convergence of the sequence generated by the proposed iterative method to a fixed point of the mapping, which is the unique solution of a certain variational inequality. In particular, we utilize weaker control conditions than previous ones in order to show strong convergence. Our results complement, develop, and improve upon the corresponding ones given by some authors recently in this area.
\end{abstract}

MSC: 47H09; 47H05; 47H10; 47J25; 49M05; 47J05

Keywords: composite iterative method; $k$-strictly pseudocontractive mapping; nonexpansive mapping; fixed points; Lipschitzian; weakly asymptotically regular; $\rho$-Lipschitzian and $\eta$-strongly monotone operator; strongly positive bounded linear operator; Hilbert space; variational inequality

\section{Introduction}

Let $H$ be a real Hilbert space with inner product $\langle\cdot, \cdot\rangle$ and induced norm $\|\cdot\|$. Let $C$ be a nonempty closed convex subset of $H$ and let $T: C \rightarrow C$ be a self-mapping on $C$. We denote by $\operatorname{Fix}(T)$ the set of fixed points of $T$.

We recall that a mapping $T: C \rightarrow H$ is said to be $k$-strictly pseudocontractive if there exists a constant $k \in[0,1)$ such that

$$
\|T x-T y\|^{2} \leq\|x-y\|^{2}+k\|(I-T) x-(I-T) y\|^{2}, \quad \forall x, y \in C .
$$

The mapping $T$ is pseudocontractive if and only if

$$
\langle T x-T y, x-y\rangle \leq\|x-y\|^{2}, \quad \forall x, y \in C .
$$

$T$ is strongly pseudocontractive if and only if there exists a constant $\lambda \in(0,1)$ such that

$$
\langle T x-T y, x-y\rangle \leq(1-\lambda)\|x-y\|^{2}, \quad \forall x, y \in C .
$$

Note that the class of $k$-strictly pseudocontractive mappings includes the class of nonexpansive mappings $T$ on $C$ (i.e., $\|T x-T y\| \leq\|x-y\|, \forall x, y \in C$ ) as a subclass. That is, $T$ is nonexpansive if and only if $T$ is 0 -strictly pseudocontractive. The mapping $T$ is also said

○2014 Jung; licensee Springer. This is an Open Access article distributed under the terms of the Creative Commons Attribution License (http://creativecommons.org/licenses/by/2.0), which permits unrestricted use, distribution, and reproduction in any medium, provided the original work is properly cited. 
to be pseudocontractive if $k=1$ and $T$ is said to be strongly pseudocontractive if there exists a positive constant $\lambda \in(0,1)$ such that $T-\lambda I$ is pseudocontractive. Clearly, the class of $k$-strictly pseudocontractive mappings falls into the one between classes of nonexpansive mappings and pseudocontractive mappings. Also we remark that the class of strongly pseudocontractive mappings is independent of the class of $k$-strictly pseudocontractive mappings (see [1-3]). The class of pseudocontractive mappings is one of the most important classes of mappings among nonlinear mappings. Recently, many authors have been devoting the studies on the problems of finding fixed points for pseudocontractive mappings; see, for example, [4-9] and the references therein.

Let $A$ be a strongly positive bounded linear operator on $H$. That is, there is a constant $\bar{\gamma}>0$ with the property

$$
\langle A x, x\rangle \geq \bar{\gamma}\|x\|^{2}, \quad \forall x \in H
$$

It is well known that iterative methods for nonexpansive mappings can be used to solve a convex minimization problem: see, e.g., [10-12] and the references therein. A typical problem is that of minimizing a quadratic function over the set of fixed points of a nonexpansive mapping on a real Hilbert space $H$ :

$$
\min _{x \in C} \frac{1}{2}\langle A x, x\rangle-\langle x, b\rangle
$$

where $C$ is the fixed point set of a nonexpansive mapping $S$ on $H$ and $b$ is a given point in $H$. In [11], Xu proved that the sequence $\left\{x_{n}\right\}$ generated by the iterative method for a nonexpansive mapping $S$ presented below with the initial guess $x_{0} \in H$ chosen arbitrary:

$$
x_{n+1}=\alpha_{n} b+\left(I-\alpha_{n} A\right) S x_{n}, \quad \forall n \geq 0,
$$

converges strongly to the unique solution of the minimization problem (1.1) provided the sequence $\left\{\alpha_{n}\right\}$ satisfies certain conditions.

In [13], combining the Moudafi viscosity approximation method [14] with Xu's method (1.2), Marino and $\mathrm{Xu}$ [13] considered the following general iterative method for a nonexpansive mapping $S$ :

$$
x_{n+1}=\alpha_{n} \gamma f x_{n}+\left(I-\alpha_{n} A\right) S x_{n}, \quad \forall n \geq 0,
$$

where $f$ is a contractive mapping on $H$ with a constant $\alpha \in(0,1)$ (i.e., there exists a constant $\alpha \in(0,1)$ such that $\|f(x)-f(y)\| \leq \alpha\|x-y\|, \forall x, y \in H)$. They proved that if the sequence $\left\{\alpha_{n}\right\}$ of control parameters satisfies appropriate conditions, then the sequence $\left\{x_{n}\right\}$ generated by (1.3) converges strongly to the unique solution of the variational inequality

$$
\langle(\gamma f-A) \widetilde{x}, x-\widetilde{x}\rangle \leq 0, \quad \forall x \in \operatorname{Fix}(S),
$$

which is the optimality condition for the minimization problem

$$
\min _{x \in \operatorname{Fix}(S)} \frac{1}{2}\langle A x, x\rangle-h(x)
$$

where $h$ is a potential function for $\gamma f\left(\right.$ i.e., $\left.h^{\prime}=\gamma f\right)$. 
On the other hand, Yamada [12] introduced the following hybrid steepest-descent method for a nonexpansive mapping $S$ for solving the variational inequality:

$$
x_{n+1}=\left(I-\xi_{n} \mu F\right) S x_{n}, \quad \forall n \geq 0,
$$

where $S: H \rightarrow H$ is a nonexpansive mapping with $\operatorname{Fix}(S) \neq \emptyset ; F: H \rightarrow H$ is a $\rho$ Lipschitzian and $\eta$-strongly monotone operator with constants $\rho>0$ and $\eta>0$ (i.e., $\|F x-F y\| \leq \rho\|x-y\|$ and $\langle F x-F y, x-y\rangle \geq \eta\|x-y\|^{2}, x, y \in H$, respectively), and $0<\mu<\frac{2 \eta}{\rho^{2}}$, and then proved that if $\left\{\xi_{n}\right\}$ satisfies appropriate conditions, the sequence $\left\{x_{n}\right\}$ generated by (1.4) converges strongly to the unique solution of the variational inequality:

$$
\langle F \widetilde{x}, x-\widetilde{x}\rangle \geq 0, \quad \forall x \in \operatorname{Fix}(S) .
$$

In 2010, by combining Yamada's hybrid steepest-descent method (1.4) with Marino with Xu's method (1.3), Tian [15] introduced the following general iterative method for a nonexpansive mapping $S$ :

$$
x_{n+1}=\alpha_{n} \gamma f x_{n}+\left(I-\alpha_{n} \mu F\right) S x_{n}, \quad \forall n \geq 0,
$$

where $f$ is a contractive mapping on $H$ with a constant $\alpha \in(0,1)$. His results improved and complemented the corresponding results of Marino and $\mathrm{Xu}$ [13]. In [16], Tian also considered the following general iterative method for a nonexpansive mapping $S$ :

$$
x_{n+1}=\alpha_{n} \gamma V x_{n}+\left(I-\alpha_{n} \mu F\right) S x_{n}, \quad \forall n \geq 0,
$$

where $V: H \rightarrow H$ is a Lipschitzian mapping with a constant $l \geq 0$. In particular, the results in [16] extended the results of Tian [15] from the case of the contractive mapping $f$ to the case of a Lipschitzian mapping $V$.

In 2011, Ceng et al. [17] also introduced the following iterative method for the nonexpansive mapping $S$ :

$$
x_{n+1}=P_{C}\left[\alpha_{n} \gamma V x_{n}+\left(I-\alpha_{n} \mu F\right) S x_{n}\right], \quad \forall n \geq 0,
$$

where $F: C \rightarrow H$ is a $\rho$-Lipschitzian and $\eta$-strongly monotone operator with constants $\rho>0$ and $\eta>0, V: C \rightarrow H$ is an $l$-Lipschitzian mapping with a constant $l \geq 0$ and $0<$ $\mu<\frac{2 \eta}{\rho^{2}}$. In particular, by using appropriate control conditions on $\left\{\alpha_{n}\right\}$, they proved that the sequence $\left\{x_{n}\right\}$ generated by (1.7) converges strongly to a fixed point $\tilde{x}$ of $S$, which is the unique solution of the following variational inequality related to the operator $F$ :

$$
\langle\mu F \widetilde{x}-\gamma V \widetilde{x}, \widetilde{x}-p\rangle \leq 0, \quad \forall p \in \operatorname{Fix}(S) .
$$

Their results also improved the results of Tian [15] from the case of the contractive mapping $f$ to the case of a Lipschitzian mapping $V$.

In 2011, Ceng et al. [18] introduced the following general composite iterative method for a nonexpansive mapping $S$ :

$$
\left\{\begin{array}{l}
y_{n}=\left(I-\alpha_{n} \mu F\right) S x_{n}+\alpha_{n} \gamma f x_{n}, \\
x_{n+1}=\left(I-\beta_{n} A\right) S x_{n}+\beta_{n} y_{n}, \quad \forall n \geq 0,
\end{array}\right.
$$


which combines Xu's method (1.2) with Tian's method (1.5). Under appropriate control conditions on $\left\{\alpha_{n}\right\}$ and $\left\{\beta_{n}\right\}$, they proved that the sequence $\left\{x_{n}\right\}$ generated by (1.8) converges strongly to a fixed point $\tilde{x}$ of $S$, which is the unique solution of the following variational inequality related to the operator $A$ :

$$
\langle(A-I) \widetilde{x}, \tilde{x}-p\rangle \leq 0, \quad \forall p \in \operatorname{Fix}(S) .
$$

Their results supplemented and developed the corresponding ones of Marino and Xu [13], Yamada [12] and Tian [15].

On the another hand, in 2011, by combining Yamada's hybrid steepest-descent method (1.4) with Marino and Xu's method (1.3), Jung [7] considered the following explicit iterative scheme for finding fixed points of a $k$-strictly pseudocontractive mapping $T$ for some $0 \leq$ $k<1$ :

$$
x_{n+1}=\alpha_{n} \gamma f\left(x_{n}\right)+\beta_{n} x_{n}+\left(\left(1-\beta_{n}\right) I-\alpha_{n} \mu F\right) P_{C} S x_{n}, \quad \forall n \geq 0,
$$

where $S: C \rightarrow H$ is a mapping defined by $S x=k x+(1-k) T x ; P_{C}$ is the metric projection of $H$ onto $C$; $f: C \rightarrow C$ is a contractive mapping with a constant $\alpha \in(0,1) ; F: C \rightarrow C$ is a $\rho$-Lipschitzian and $\eta$-strongly monotone operator with constants $\rho>0$ and $\eta>0$; and $0<\mu<\frac{2 \eta}{\rho^{2}}$. Under suitable control conditions on $\left\{\alpha_{n}\right\}$ and $\left\{\beta_{n}\right\}$, he proved that the sequence $\left\{x_{n}\right\}$ generated by (1.9) converges strongly to a fixed point $\tilde{x}$ of $T$, which is the unique solution of the following variational inequality related to the operator $F$ :

$$
\langle\mu F \tilde{x}-\gamma f \widetilde{x}, \tilde{x}-p\rangle \leq 0, \quad \forall p \in \operatorname{Fix}(T) .
$$

His result also improved and complemented the corresponding results of Cho et al. [5], Jung [6], Marino and Xu [13] and Tian [15].

In this paper, motivated and inspired by the above-mentioned results, we will combine Xu's method (1.2) with Tian's method (1.6) for a $k$-strictly pseudocontractive mapping $T$ for some $0 \leq k<1$ and consider the following new general composite iterative method for finding an element of $\operatorname{Fix}(T)$ :

$$
\left\{\begin{array}{l}
y_{n}=\alpha_{n} \gamma V x_{n}+\left(I-\alpha_{n} \mu F\right) T_{n} x_{n}, \\
x_{n+1}=\left(I-\beta_{n} A\right) T_{n} x_{n}+\beta_{n} y_{n}, \quad \forall n \geq 0,
\end{array}\right.
$$

where $T_{n}: H \rightarrow H$ is a mapping defined by $T_{n} x=\lambda_{n} x+\left(1-\lambda_{n}\right) T x$ for $0 \leq k \leq \lambda_{n} \leq \lambda<1$ and $\lim _{n \rightarrow \infty} \lambda_{n}=\lambda ; A$ is a strongly positive bounded linear operator on $H$ with a constant $\bar{\gamma} \in(1,2) ;\left\{\alpha_{n}\right\} \subset[0,1]$ and $\left\{\beta_{n}\right\} \subset(0,1]$ satisfy appropriate conditions; $V: H \rightarrow H$ is a Lipschitzian mapping with a constant $l \geq 0 ; F: H \rightarrow H$ is a $\rho$-Lipschitzian and $\eta$-strongly monotone operator with constants $\rho>0$ and $\eta>0$; and $0<\mu<\frac{2 \eta}{\rho^{2}}$. By using weaker control conditions than previous ones, we establish the strong convergence of the sequence generated by the proposed iterative method (1.10) to a point $\tilde{x}$ in Fix $(T)$, which is the unique solution of the variational inequality related to $A$ :

$$
\langle(A-I) \widetilde{x}, \widetilde{x}-p\rangle \leq 0, \quad \forall p \in \operatorname{Fix}(T)
$$


Our results complement, develop, and improve upon the corresponding ones given by Cho et al. [5] and Jung [6-8] for the strictly pseudocontractive mapping as well as Yamada [12], Marino and $\mathrm{Xu}$ [13], Tian [15] and Ceng et al. [17] and Ceng et al. [18] for the nonexpansive mapping.

\section{Preliminaries and lemmas}

Throughout this paper, when $\left\{x_{n}\right\}$ is a sequence in $H, x_{n} \rightarrow x$ (resp., $x_{n} \rightarrow x$ ) will denote strong (resp., weak) convergence of the sequence $\left\{x_{n}\right\}$ to $x$.

For every point $x \in H$, there exists a unique nearest point in $C$, denoted by $P_{C}(x)$, such that

$$
\left\|x-P_{C}(x)\right\| \leq\|x-y\|, \quad \forall y \in C .
$$

$P_{C}$ is called the metric projection of $H$ to $C$. It is well known that $P_{C}$ is nonexpansive and that, for $x \in H$,

$$
z=P_{C} x \quad \Longleftrightarrow \quad\langle x-z, y-z\rangle \leq 0, \quad \forall y \in C .
$$

In a Hilbert space $H$, we have

$$
\|x-y\|^{2}=\|x\|^{2}+\|y\|^{2}-2\langle x, y\rangle, \quad \forall x, y \in H .
$$

Lemma 2.1 In a real Hilbert space $H$, the following inequality holds:

$$
\|x+y\|^{2} \leq\|x\|^{2}+2\langle y, x+y\rangle, \quad \forall x, y \in H .
$$

Let LIM be a Banach limit. According to time and circumstances, we use $\operatorname{LIM}_{n}\left(a_{n}\right)$ instead of $\operatorname{LIM}(a)$ for every $a=\left\{a_{n}\right\} \in \ell^{\infty}$. The following properties are well known:

(i) for all $n \geq 1, a_{n} \leq c_{n}$ implies $\operatorname{LIM}_{n}\left(a_{n}\right) \leq \operatorname{LIM}_{n}\left(c_{n}\right)$,

(ii) $\operatorname{LIM}_{n}\left(a_{n+N}\right)=\operatorname{LIM}_{n}\left(a_{n}\right)$ for any fixed positive integer $N$,

(iii) $\liminf _{n \rightarrow \infty} a_{n} \leq \operatorname{LIM}_{n}\left(a_{n}\right) \leq \lim \sup _{n \rightarrow \infty} a_{n}$ for all $\left\{a_{n}\right\} \in l^{\infty}$.

The following lemma was given in [19, Proposition 2].

Lemma 2.2 Let $a \in \mathbb{R}$ be a real number and let a sequence $\left\{a_{n}\right\} \in l^{\infty}$ satisfy the condition $\operatorname{LIM}_{n}\left(a_{n}\right) \leq a$ for all Banach limit LIM. If $\lim _{\sup _{n \rightarrow \infty}}\left(a_{n+1}-a_{n}\right) \leq 0$, then $\lim \sup _{n \rightarrow \infty} a_{n} \leq a$.

We also need the following lemmas for the proof of our main results.

Lemma $2.3([20,21])$ Let $\left\{s_{n}\right\}$ be a sequence of non-negative real numbers satisfying

$$
s_{n+1} \leq\left(1-\omega_{n}\right) s_{n}+\omega_{n} \delta_{n}+r_{n}, \quad \forall n \geq 0,
$$

where $\left\{\omega_{n}\right\},\left\{\delta_{n}\right\}$, and $\left\{r_{n}\right\}$ satisfy the following conditions:

(i) $\left\{\omega_{n}\right\} \subset[0,1]$ and $\sum_{n=0}^{\infty} \omega_{n}=\infty$,

(ii) $\lim \sup _{n \rightarrow \infty} \delta_{n} \leq 0$ or $\sum_{n=0}^{\infty} \omega_{n}\left|\delta_{n}\right|<\infty$,

(iii) $r_{n} \geq 0(n \geq 0), \sum_{n=0}^{\infty} r_{n}<\infty$.

Then $\lim _{n \rightarrow \infty} s_{n}=0$. 
Lemma 2.4 ([22] Demiclosedness principle) Let $C$ be a nonempty closed convex subset of a real Hilbert space $H$, and let $S: C \rightarrow C$ be a nonexpansive mapping. Then the mapping $I-S$ is demiclosed. That is, if $\left\{x_{n}\right\}$ is a sequence in $C$ such that $x_{n} \rightarrow x^{*}$ and $(I-S) x_{n} \rightarrow y$, then $(I-S) x^{*}=y$.

Lemma 2.5 ([23]) Let $H$ be a real Hilbert space and let $C$ be a closed convex subset of $H$. Let $T: C \rightarrow H$ be a $k$-strictly pseudocontractive mapping on $C$. Then the following hold:

(i) The fixed point set $\operatorname{Fix}(T)$ is closed convex, so that the projection $P_{\mathrm{Fix}(T)}$ is well defined.

(ii) $\operatorname{Fix}\left(P_{C} T\right)=\operatorname{Fix}(T)$.

(iii) If we define a mapping $S: C \rightarrow H$ by $S x=\lambda x+(1-\lambda)$ Tx for all $x \in C$. then, as $\lambda \in[k, 1), S$ is a nonexpansive mapping such that $\operatorname{Fix}(T)=\operatorname{Fix}(S)$.

The following lemma can easily be proven (see also [12]).

Lemma 2.6 Let $H$ be a real Hilbert space $H$. Let $F: H \rightarrow H$ be a $\rho$-Lipschitzian and $\eta$ strongly monotone operator with constants $\rho>0$ and $\eta>0$. Let $0<\mu<\frac{2 \eta}{\rho^{2}}$ and $0<t<$ $\xi \leq 1$. Then $G:=\xi I-t \mu F: H \rightarrow H$ is a contractive mapping with constant $\xi-t \tau$, where $\tau=1-\sqrt{1-\mu\left(2 \eta-\mu \rho^{2}\right)}$.

Lemma 2.7 ([13]) Assume that $A$ is a strongly positive bounded linear operator on $H$ with a coefficient $\bar{\gamma}>0$ and $0<\zeta \leq\|A\|^{-1}$. Then $\|I-\zeta A\| \leq 1-\zeta \bar{\gamma}$.

Finally, we recall that the sequence $\left\{x_{n}\right\}$ in $H$ is said to be weakly asymptotically regular if

$$
w-\lim _{n \rightarrow \infty}\left(x_{n+1}-x_{n}\right)=0, \quad \text { that is, } x_{n+1}-x_{n} \rightarrow 0
$$

and asymptotically regular if

$$
\lim _{n \rightarrow \infty}\left\|x_{n+1}-x_{n}\right\|=0
$$

respectively.

\section{The main results}

Throughout the rest of this paper, we always assume the following:

- $H$ is a real Hilbert space;

- $T: H \rightarrow H$ is a $k$-strictly pseudocontractive mapping with $\operatorname{Fix}(T) \neq \emptyset$ for some $0 \leq k<1$;

- $F: H \rightarrow H$ is a $\rho$-Lipschitzian and $\eta$-strongly monotone operator with constants $\rho>0$ and $\eta>0$;

- $A: H \rightarrow H$ is a strongly positive linear bounded operator on $H$ with a constant $\bar{\gamma} \in(1,2)$;

- $V: H \rightarrow H$ is an $l$-Lipschitzian mapping with a constant $l \geq 0$;

- $0<\mu<\frac{2 \eta}{\rho^{2}}$ and $0 \leq \gamma l<\tau$, where $\tau=1-\sqrt{1-\mu\left(2 \eta-\mu \rho^{2}\right)}$;

- $T_{t}: H \rightarrow H$ is a mapping defined by $T_{t} x=\lambda_{t} x+\left(1-\lambda_{t}\right) T x, t \in(0,1)$, for $0 \leq k \leq \lambda_{t} \leq \lambda<1$ and $\lim _{t \rightarrow 0} \lambda_{t}=\lambda$;

- $T_{n}: H \rightarrow H$ is a mapping defined by $T_{n} x=\lambda_{n} x+\left(1-\lambda_{n}\right) T x$ for $0 \leq k \leq \lambda_{n} \leq \lambda<1$ and $\lim _{n \rightarrow \infty} \lambda_{n}=\lambda$;

- $P_{\mathrm{Fix}(T)}$ is a metric projection of $H$ onto $\operatorname{Fix}(T)$. 
By Lemma 2.5(iii), we note that $T_{t}$ and $T_{n}$ are nonexpansive and $\operatorname{Fix}(T)=\operatorname{Fix}\left(T_{t}\right)=$ $\operatorname{Fix}\left(T_{n}\right)$.

In this section, we introduce the following general composite scheme that generates a net $\left\{x_{t}\right\}_{t \in\left(0, \min \left\{1, \frac{2-\bar{\gamma}}{\tau-\gamma l}\right\}\right)}$ in an implicit way:

$$
x_{t}=\left(I-\theta_{t} A\right) T_{t} x_{t}+\theta_{t}\left[t \gamma V x_{t}+(I-t \mu F) T_{t} x_{t}\right] .
$$

We prove strong convergence of $\left\{x_{t}\right\}$ as $t \rightarrow 0$ to a fixed point $\tilde{x}$ of $T$ which is a solution of the following variational inequality:

$$
\langle(A-I) \tilde{x}, \tilde{x}-p\rangle \leq 0, \quad \forall p \in \operatorname{Fix}(T)
$$

We also propose the following general composite explicit scheme, which generates a sequence in an explicit way:

$$
\left\{\begin{array}{l}
y_{n}=\alpha_{n} \gamma V x_{n}+\left(I-\alpha_{n} \mu F\right) T_{n} x_{n}, \\
x_{n+1}=\left(I-\beta_{n} A\right) T_{n} x_{n}+\beta_{n} y_{n}, \quad \forall n \geq 0,
\end{array}\right.
$$

where $\left\{\alpha_{n}\right\} \in[0,1],\left\{\beta_{n}\right\} \subset(0,1]$ and $x_{0} \in H$ is an arbitrary initial guess, and establish strong convergence of this sequence to a fixed point $\tilde{x}$ of $T$, which is also the unique solution of the variational inequality (3.2).

Now, for $t \in\left(0, \min \left\{1, \frac{2-\bar{\gamma}}{\tau-\gamma l}\right\}\right)$ and $\theta_{t} \in\left(0,\|A\|^{-1}\right]$, consider a mapping $Q_{t}: H \rightarrow H$ defined by

$$
Q_{t} x=\left(I-\theta_{t} A\right) T_{t} x+\theta_{t}\left[t \gamma V x+(I-t \mu F) T_{t} x\right], \quad \forall x \in H
$$

It is easy to see that $Q_{t}$ is a contractive mapping with constant $1-\theta_{t}(\bar{\gamma}-1+t(\tau-\gamma l))$. Indeed, by Lemma 2.6 and Lemma 2.7 , we have

$$
\begin{aligned}
\left\|Q_{t} x-Q_{t} y\right\| \leq & \left\|\left(I-\theta_{t} A\right) T_{t} x-\left(I-\theta_{t} A\right) T_{t} y\right\| \\
& +\theta_{t}\left\|\left[t \gamma V x+(I-t \mu F) T_{t} x\right]-\left[t \gamma V y+(I-t \mu F) T_{t} y\right]\right\| \\
\leq & \left(1-\theta_{t} \bar{\gamma}\right)\|x-y\|+\theta_{t}\left[t \gamma\|V x-V y\|+\left\|(I-t \mu F) T_{t} x-(I-t \mu F) T_{t} y\right\|\right] \\
\leq & \left(1-\theta_{t} \bar{\gamma}\right)\|x-y\|+\theta_{t}(1-t(\tau-\gamma l))\|x-y\| \\
= & {\left[1-\theta_{t}(\bar{\gamma}-1+t(\tau-\gamma l))\right]\|x-y\| . }
\end{aligned}
$$

Since $\bar{\gamma} \in(1,2), \tau-\gamma l>0$, and

$$
0<t<\min \left\{1, \frac{2-\bar{\gamma}}{\tau-\gamma l}\right\} \leq \frac{2-\bar{\gamma}}{\tau-\gamma l}
$$

it follows that

$$
0<(\bar{\gamma}-1+t(\tau-\gamma l))<1,
$$


which along with $0<\theta_{t} \leq\|A\|^{-1}<1$ yields

$$
0<1-\theta_{t}(\bar{\gamma}-1+t(\tau-\gamma l))<1 .
$$

Hence $Q_{t}$ is a contractive mapping. By the Banach contraction principle, $Q_{t}$ has a unique fixed point, denoted $x_{t}$, which uniquely solves the fixed point equation (3.1).

We summary the basic properties of $\left\{x_{t}\right\}$, which can be proved by the same method in [18]. We include its proof for the sake of completeness.

Proposition 3.1 Let $\left\{x_{t}\right\}$ be defined via (3.1). Then

(i) $\left\{x_{t}\right\}$ is bounded for $t \in\left(0, \min \left\{1, \frac{2-\bar{\gamma}}{\tau-\gamma l}\right\}\right)$;

(ii) $\lim _{t \rightarrow 0}\left\|x_{t}-T_{t} x_{t}\right\|=0$ provided $\lim _{t \rightarrow 0} \theta_{t}=0$;

(iii) $x_{t}:\left(0, \min \left\{1, \frac{2-\bar{\gamma}}{\tau-\gamma l}\right\}\right) \rightarrow H$ is locally Lipschitzian provided $\theta_{t}:\left(0, \min \left\{1, \frac{2-\bar{\gamma}}{\tau-\gamma l}\right\}\right) \rightarrow$ $\left(0,\|A\|^{-1}\right]$ is locally Lipschitzian, and $\lambda_{t}:\left(0, \min \left\{1, \frac{2-\bar{\gamma}}{\tau-\gamma l}\right\}\right) \rightarrow[k, \lambda]$ is locally Lipschitzian;

(iv) $x_{t}$ defines a continuous path from $\left(0, \min \left\{1, \frac{2-\bar{\gamma}}{\tau-\gamma l}\right\}\right)$ into $H$ provided

$\theta_{t}:\left(0, \min \left\{1, \frac{2-\bar{\gamma}}{\tau-\gamma l}\right\}\right) \rightarrow\left(0,\|A\|^{-1}\right]$ is continuous, and $\lambda_{t}:\left(0, \min \left\{1, \frac{2-\bar{\gamma}}{\tau-\gamma l}\right\}\right) \rightarrow[k, \lambda]$ is continuous.

Proof (1) Let $p \in \operatorname{Fix}(T)$. Observing $\operatorname{Fix}(T)=\operatorname{Fix}\left(T_{t}\right)$ by Lemma 2.5(iii), we have

$$
\begin{aligned}
&\left\|x_{t}-p\right\| \\
&=\left\|\left(I-\theta_{t} A\right) T_{t} x_{t}+\theta_{t}\left[t \gamma V x_{t}+(I-t \mu F) T_{t} x_{t}\right]-p\right\| \\
&=\left\|\left(I-\theta_{t} A\right) T_{t} x_{t}-\left(I-\theta_{t} A\right) T_{t} p+\theta_{t}\left[t \gamma V x_{t}+(I-t \mu F) T_{t} x_{t}-p\right]+\theta_{t}(I-A) p\right\| \\
& \leq\left\|\left(I-\theta_{t} A\right) T_{t} x_{t}-\left(I-\theta_{t} A\right) T_{t} p\right\|+\theta_{t}\left\|t \gamma V x_{t}+(I-t \mu F) T_{t} x_{t}-p\right\|+\theta_{t}\|(I-A) p\| \\
&=\left\|\left(I-\theta_{t} A\right) T_{t} x_{t}-\left(I-\theta_{t} A\right) T_{t} p\right\| \\
&+\theta_{t}\left\|(I-t \mu F) T_{t} x_{t}-(I-t \mu F) T_{t} p+t\left(\gamma V x_{t}-\mu F p\right)\right\|+\theta_{t}\|I-A\|\|p\| \\
& \leq\left(1-\theta_{t} \bar{\gamma}\right)\left\|x_{t}-p\right\|+\theta_{t}\left[(1-t \tau)\left\|x_{t}-p\right\|+t\left(\gamma l\left\|x_{t}-p\right\|+\|\gamma V p-\mu F p\|\right)\right] \\
&+\theta_{t}\|I-A\|\|p\| .
\end{aligned}
$$

So, it follows that

$$
\begin{aligned}
\left\|x_{t}-p\right\| & \leq \frac{\|I-A\|\|p\|+t\|\gamma V p-\mu F p\|}{\bar{\gamma}-1+t(\tau-\gamma l)} \leq \frac{\|I-A\|\|p\|+t\|\gamma V p-\mu F p\|}{\bar{\gamma}-1} \\
& \leq \frac{\|I-A\|\|p\|+\|\gamma V p-\mu F p\|}{\bar{\gamma}-1} .
\end{aligned}
$$

Hence $\left\{x_{t}\right\}$ is bounded and so are $\left\{V x_{t}\right\},\left\{T x_{t}\right\},\left\{T_{t} x_{t}\right\}$, and $\left\{F T_{t} x_{t}\right\}$.

(ii) By the definition of $\left\{x_{t}\right\}$, we have

$$
\begin{aligned}
\left\|x_{t}-T_{t} x_{t}\right\| & =\left\|\theta_{t}\left[(I-A) T_{t} x_{t}+t\left(\gamma V x_{t}-\mu F T_{t} x_{t}\right)\right]\right\| \\
& =\theta_{t}\left\|(I-A) T_{t} x_{t}+t\left(\gamma V x_{t}-\mu F T_{t} x_{t}\right)\right\| \\
& \leq \theta_{t}\|I-A\|\left\|T_{t} x_{t}\right\|+t\left\|\gamma V x_{t}-\mu F T_{t} x_{t}\right\| \rightarrow 0 \quad \text { as } t \rightarrow 0,
\end{aligned}
$$

by the boundedness of $\left\{V x_{t}\right\}$ and $\left\{F T_{t} x_{t}\right\}$ in (i). 
(iii) Let $t, t_{0} \in\left(0, \min \left\{1, \frac{2-\bar{\gamma}}{\tau-\gamma l}\right\}\right)$, Noting that

$$
\begin{aligned}
\left\|T_{t} x_{t}-T_{t_{0}} x_{t_{0}}\right\| & \leq\left\|T_{t} x_{t}-T_{t} x_{t_{0}}\right\|+\left\|T_{t} x_{t_{0}}-T_{t_{0}} x_{t_{0}}\right\| \\
& \leq\left\|x_{t}-x_{t_{0}}\right\|+\left|\lambda_{t}-\lambda_{t_{0}}\right|\left\|x_{t_{0}}-T x_{t_{0}}\right\|
\end{aligned}
$$

we calculate

$$
\begin{aligned}
\| x_{t}- & x_{t_{0}} \| \\
= & \|\left(I-\theta_{t} A\right) T_{t} x_{t}+\theta_{t}\left[t \gamma V x_{t}+(I-t \mu F) T_{t} x_{t}\right] \\
& -\left(I-\theta_{t_{0}} A\right) T_{t_{0}} x_{t_{0}}-\theta_{t_{0}}\left[t_{0} \gamma V x_{t_{0}}+\left(I-t_{0} \mu F\right) T_{t_{0}} x_{t_{0}}\right] \| \\
\leq & \left\|\left(I-\theta_{t} A\right) T_{t} x_{t}-\left(I-\theta_{t_{0}} A\right) T_{t} x_{t}\right\|+\left\|\left(I-\theta_{t_{0}} A\right) T_{t} x_{t}-\left(I-\theta_{t_{0}} A\right) T_{t_{0}} x_{t_{0}}\right\| \\
& +\left|\theta_{t}-\theta_{t_{0}}\right|\left\|t \gamma V x_{t}+(I-t \mu F) T_{t} x_{t}\right\| \\
& +\theta_{t_{0}}\left\|\left[t \gamma V x_{t}+(I-t \mu F) T_{t} x_{t}\right]-\left[t_{0} \gamma V x_{t_{0}}+\left(I-t_{0} \mu F\right) T_{t_{0}} x_{t_{0}}\right]\right\| \\
\leq & \left|\theta_{t}-\theta_{t_{0}}\right|\|A\|\left\|T_{t} x_{t}\right\|+\left(1-\theta_{t_{0}} \bar{\gamma}\right)\left\|T_{t} x_{t}-T_{t_{0}} x_{t_{0}}\right\| \\
& +\left|\theta_{t}-\theta_{t_{0}}\right|\left\|t \gamma V x_{t}+(I-t \mu F) T_{t} x_{t}\right\|+\theta_{t_{0}} \|\left(t-t_{0}\right) \gamma V x_{t}+t_{0} \gamma\left(V x_{t}-V x_{t_{0}}\right) \\
& -\left(t-t_{0}\right) \mu F T_{t} x_{t}+\left(I-t_{0} \mu F\right) T_{t} x_{t}-\left(I-t_{0} \mu F\right) T_{t_{0}} x_{t_{0}} \| \\
\leq & \left|\theta_{t}-\theta_{t_{0}}\right|\|A\|\left\|T_{t} x_{t}\right\|+\left(1-\theta_{t_{0}} \bar{\gamma}\right)\left[\left\|x_{t}-x_{t_{0}}\right\|+\left|\lambda_{t}-\lambda_{t_{0}}\right|\left\|x_{t_{0}}-T x_{t_{0}}\right\|\right] \\
& +\left|\theta_{t}-\theta_{t_{0}}\right|\left[\left\|T_{t} x_{t}\right\|+t\left(\gamma\left\|V x_{t}\right\|+\mu\left\|F T_{t} x_{t}\right\|\right)\right] \\
& +\theta_{t_{0}}\left[\left(\gamma\left\|V x_{t}\right\|+\mu\left\|F T_{t} x_{t}\right\|\right)\left|t-t_{0}\right|+t_{0} \gamma l\left\|x_{t}-x_{t_{0}}\right\|+\left(1-t_{0} \tau\right)\left\|T_{t} x_{t}-T_{t_{0}} x_{t_{0}}\right\|\right] \\
\leq & \left|\theta_{t}-\theta_{t_{0}}\right|\|A\|\left\|T_{t} x_{t}\right\|+\left(1-\theta_{t_{0}} \bar{\gamma}\right)\left(\left\|x_{t}-x_{t_{0}}\right\|+\left|\lambda_{t}-\lambda_{t_{0}}\right|\left\|x_{t_{0}}-T x_{t_{0}}\right\|\right) \\
& +\left|\theta_{t}-\theta_{t_{0}}\right|\left(\left\|T_{t} x_{t}\right\|+\gamma\left\|V x_{t}\right\|+\mu\left\|F T_{t} x_{t}\right\|\right) \\
& +\theta_{t_{0}}\left(\gamma\left\|V x_{t}\right\|+\mu\left\|F T_{t} x_{t}\right\|\right)\left|t-t_{0}\right|+\theta_{t_{0}} t_{0} \gamma l\left\|x_{t}-x_{t_{0}}\right\| \\
& +\theta_{t_{0}}\left(1-t_{0} \tau\right)\left(\left\|x_{t}-x_{t_{0}}\right\|+\left|\lambda_{t}-\lambda t_{t_{0}}\right|\left\|x_{t_{0}}-T x_{t_{0}}\right\|\right) . \\
&
\end{aligned}
$$

This implies that

$$
\begin{aligned}
\left\|x_{t}-x_{t_{0}}\right\| \leq & \frac{\|A\|\left\|T_{t} x_{t}\right\|+\left\|T_{t} x_{t}\right\|+\gamma\left\|V x_{t}\right\|+\mu\left\|F T_{t} x_{t}\right\|}{\theta_{t_{0}}\left(\bar{\gamma}-1+t_{0}(\tau-\gamma l)\right)}\left|\theta_{t}-\theta_{t_{0}}\right| \\
& +\frac{\gamma\left\|V x_{t}\right\|+\mu\left\|F T_{t} x_{t}\right\|}{\bar{\gamma}-1+t_{0}(\tau-\gamma l)}\left|t-t_{0}\right| \\
& +\frac{\left[1-\theta_{t_{0}}\left(\bar{\gamma}-1+t_{0} \tau\right)\right]\left\|x_{t_{0}}-T x_{t_{0}}\right\|}{\theta_{t_{0}}\left(\bar{\gamma}-1+t_{0}(\tau-\gamma l)\right)}\left|\lambda_{t}-\lambda_{t_{0}}\right| .
\end{aligned}
$$

Since $\theta_{t}:\left(0, \min \left\{1, \frac{2-\bar{\gamma}}{\tau-\gamma l}\right\}\right) \rightarrow\left(0,\|A\|^{-1}\right]$ is locally Lipschitzian, and $\lambda_{t}:\left(0, \min \left\{1, \frac{2-\bar{\gamma}}{\tau-\gamma l}\right\}\right) \rightarrow$ $[k, \lambda]$ is locally Lipschitzian, $x_{t}$ is also locally Lipschitzian.

(iv) From the last inequality in (iii), the result follows immediately.

We prove the following theorem for strong convergence of the net $\left\{x_{t}\right\}$ as $t \rightarrow 0$, which guarantees the existence of solutions of the variational inequality (3.2). 
Theorem 3.1 Let the net $\left\{x_{t}\right\}$ be defined via (3.1). If $\lim _{t \rightarrow 0} \theta_{t}=0$, then $x_{t}$ converges strongly to a fixed point $\tilde{x}$ of $T$ as $t \rightarrow 0$, which solves the variational inequality (3.2). Equivalently, we have $P_{\mathrm{Fix}(T)}(2 I-A) \tilde{x}=\tilde{x}$.

Proof We first show the uniqueness of a solution of the variational inequality (3.2), which is indeed a consequence of the strong monotonicity of $A-I$. In fact, since $A$ is a strongly positive bounded linear operator with a coefficient $\bar{\gamma} \in(1,2)$, we know that $A-I$ is strongly monotone with a coefficient $\bar{\gamma}-1 \in(0,1)$. Suppose that $\tilde{x} \in \operatorname{Fix}(T)$ and $\widehat{x} \in \operatorname{Fix}(T)$ both are solutions to (3.2). Then we have

$$
\langle(A-I) \widetilde{x}, \tilde{x}-\widehat{x}\rangle \leq 0
$$

and

$$
\langle(A-I) \widehat{x}, \widehat{x}-\tilde{x}\rangle \leq 0 .
$$

Adding up (3.4) and (3.5) yields

$$
\langle(A-I) \tilde{x}-(A-I) \widehat{x}, \tilde{x}-\widehat{x}\rangle \leq 0 .
$$

The strong monotonicity of $A-I$ implies that $\widetilde{x}=\widehat{x}$ and the uniqueness is proved.

Next, we prove that $x_{t} \rightarrow \tilde{x}$ as $t \rightarrow 0$. Observing Fix $(T)=$ Fix $\left(T_{t}\right)$ by Lemma 2.5(iii), from (3.1), we write, for given $p \in \operatorname{Fix}(T)$,

$$
\begin{aligned}
x_{t}-p= & \left(I-\theta_{t} A\right) T_{t} x_{t}-\left(I-\theta_{t} A\right) T_{t} p+\theta_{t}\left[t \gamma V x_{t}+(I-t \mu F) T_{t} x_{t}-p\right]+\theta_{t}(I-A) p \\
= & \left(I-\theta_{t} A\right)\left(T_{t} x_{t}-T_{t} p\right)+\theta_{t}\left[t\left(\gamma V x_{t}-\mu F p\right)+(I-t \mu F) T_{t} x_{t}-(I-t \mu F) p\right] \\
& +\theta_{t}(I-A) p,
\end{aligned}
$$

to derive

$$
\begin{aligned}
\left\|x_{t}-p\right\|^{2}= & \left\langle\left(I-\theta_{t} A\right)\left(T_{t} x_{t}-T_{t} p\right), x_{t}-p\right\rangle+\theta_{t}\left[t\left\langle\gamma V x_{t}-\mu F p, x_{t}-p\right\rangle\right. \\
& \left.+\left\langle(I-t \mu F) T_{t} x_{t}-(I-t \mu F) p, x_{t}-p\right\rangle\right]+\theta_{t}\left\langle(I-A) p, x_{t}-p\right\rangle \\
\leq & \left(1-\theta_{t} \bar{\gamma}\right)\left\|x_{t}-p\right\|^{2}+\theta_{t}\left[(1-t \tau)\left\|x_{t}-p\right\|^{2}+t \gamma l\left\|x_{t}-p\right\|^{2}\right. \\
& \left.+t\left\langle\gamma V p-\mu F p, x_{t}-p\right\rangle\right]+\theta_{t}\left((I-A) p, x_{t}-p\right\rangle \\
= & {\left[1-\theta_{t}(\bar{\gamma}-1+t(\tau-\gamma l))\right]\left\|x_{t}-p\right\|^{2} } \\
& +\theta_{t}\left(t\left\langle\gamma V p-\mu F p, x_{t}-p\right\rangle+\left\langle(I-A) p, x_{t}-p\right\rangle\right) .
\end{aligned}
$$

Therefore,

$$
\left\|x_{t}-p\right\|^{2} \leq \frac{1}{\bar{\gamma}-1+t(\tau-\gamma l)}\left(t\left\langle\gamma V p-\mu F p, x_{t}-p\right\rangle+\left\langle(I-A) p, x_{t}-p\right\rangle\right) .
$$

Since $\left\{x_{t}\right\}$ is bounded as $t \rightarrow 0$ (by Proposition 3.1(i)), we see that if $\left\{t_{n}\right\}$ is a subsequence in $\left(0, \min \left\{1, \frac{2-\bar{\gamma}}{\tau-\gamma l}\right\}\right)$ such that $t_{n} \rightarrow 0$ and $x_{t_{n}} \rightarrow x^{*}$, then from (3.6), we obtain $x_{t_{n}} \rightarrow x^{*}$. We 
show that $x^{*} \in \operatorname{Fix}(T)$. To this end, define $S: H \rightarrow H$ by $S x=\lambda x+(1-\lambda) T x, \forall x \in H$, for $0 \leq k \leq \lambda<1$. Then $S$ is nonexpansive with $\operatorname{Fix}(S)=\operatorname{Fix}(T)$ by Lemma 2.5(iii). Noticing that

$$
\begin{aligned}
\left\|S x_{t_{n}}-x_{t_{n}}\right\| & \leq\left\|S x_{t_{n}}-T_{t_{n}} x_{t_{n}}\right\|+\left\|T_{t_{n}} x_{t_{n}}-x_{t_{n}}\right\| \\
& =\left(\lambda-\lambda_{t_{n}}\right)\left\|x_{t_{n}}-T x_{t_{n}}\right\|+\left\|T_{t_{n}} x_{t_{n}}-x_{t_{n}}\right\| \\
& =\frac{\lambda-\lambda_{t_{n}}}{1-\lambda_{t_{n}}}\left\|x_{t_{n}}-T_{t_{n}} x_{t_{n}}\right\|+\left\|T_{t_{n}} x_{t_{n}}-x_{t_{n}}\right\| \\
& =\frac{1+\lambda-2 \lambda_{t_{n}}}{1-\lambda_{t_{n}}}\left\|x_{t_{n}}-T_{t_{n}} x_{t_{n}}\right\|,
\end{aligned}
$$

by Proposition 3.1(ii) and $\lambda_{t_{n}} \rightarrow \lambda$ as $t_{n} \rightarrow 0$, we have $\lim _{n \rightarrow \infty}(I-S) x_{t_{n}}=0$. Thus it follows from Lemma 2.4 that $x^{*} \in \operatorname{Fix}(S)$. By Lemma 2.5(iii), we get $x^{*} \in \operatorname{Fix}(T)$.

Finally, we prove that $x^{*}$ is a solution of the variational inequality (3.2). Since

$$
x_{t}=\left(I-\theta_{t} A\right) T_{t} x_{t}+\theta_{t}\left[t \gamma V x_{t}+(I-t \mu F) T_{t} x_{t}\right]
$$

we have

$$
x_{t}-T_{t} x_{t}=\theta_{t}(I-A) T_{t} x_{t}+\theta_{t} t\left(\gamma V x_{t}-\mu F T_{t} x_{t}\right) .
$$

Since $T_{t}$ is nonexpansive, $I-T_{t}$ is monotone. So, from the monotonicity of $I-T_{t}$, it follows that, for $p \in \operatorname{Fix}(T)=\operatorname{Fix}\left(T_{t}\right)$,

$$
\begin{aligned}
0 & \leq\left\langle\left(I-T_{t}\right) x_{t}-\left(I-T_{t}\right) p, x_{t}-p\right\rangle=\left\langle\left(I-T_{t}\right) x_{t}, x_{t}-p\right\rangle \\
& =\theta_{t}\left|(I-A) T_{t} x_{t}, x_{t}-p\right\rangle+\theta_{t} t\left\langle\gamma V x_{t}-\mu F T_{t} x_{t}, x_{t}-p\right\rangle \\
& =\theta_{t}\left|(I-A) x_{t}, x_{t}-p\right\rangle+\theta_{t}\left|(I-A)\left(T_{t}-I\right) x_{t}, x_{t}-p\right\rangle+\theta_{t} t\left\langle\gamma V x_{t}-\mu F T_{t} x_{t}, x_{t}-p\right\rangle .
\end{aligned}
$$

This implies that

$$
\left\langle(A-I) x_{t}, x_{t}-p\right\rangle \leq\left\langle(I-A)\left(T_{t}-I\right) x_{t}, x_{t}-p\right\rangle+t\left\langle\gamma V x_{t}-\mu F T_{t} x_{t}, x_{t}-p\right\rangle .
$$

Now, replacing $t$ in (3.7) with $t_{n}$ and letting $n \rightarrow \infty$, noticing the boundedness of $\left\{\gamma V x_{t_{n}}-\right.$ $\left.\mu F T_{t_{n}} x_{t_{n}}\right\}$ and the fact that $(I-A)\left(T_{t_{n}}-I\right) x_{t_{n}} \rightarrow 0$ as $n \rightarrow \infty$ by Proposition 3.1(ii), we obtain

$$
\left\langle(A-I) x^{*}, x^{*}-p\right\rangle \leq 0
$$

That is, $x^{*} \in \operatorname{Fix}(T)$ is a solution of the variational inequality (3.2); hence $x^{*}=\tilde{x}$ by uniqueness. In summary, we have shown that each cluster point of $\left\{x_{t}\right\}$ (at $t \rightarrow 0$ ) equals $\widetilde{x}$. Therefore $x_{t} \rightarrow \tilde{x}$ as $t \rightarrow 0$.

The variational inequality (3.2) can be rewritten as

$$
\langle(2 I-A) \widetilde{x}-\tilde{x}, \tilde{x}-p\rangle \geq 0, \quad \forall p \in \operatorname{Fix}(T) .
$$


Recalling Lemma 2.5(i) and (2.1), this is equivalent to the fixed point equation

$$
\left.P_{\mathrm{Fix}(T)}(2 I-A) \widetilde{x}\right)=\widetilde{x} .
$$

Taking $F=I, \mu=1$ and $\gamma=1$ in Theorem 3.1, we get

Corollary 3.1 Let $\left\{x_{t}\right\}$ be defined by

$$
x_{t}=\left(I-\theta_{t} A\right) T_{t} x_{t}+\theta_{t}\left[t V x_{t}+(1-t) T_{t} x_{t}\right] .
$$

If $\lim _{t \rightarrow 0} \theta_{t}=0$, then $\left\{x_{t}\right\}$ converges strongly as $t \rightarrow 0$ to a fixed point $\tilde{x}$ of $T$, which is the unique solution of variational inequality (3.2).

First, we prove the following result in order to establish strong convergence of the sequence $\left\{x_{n}\right\}$ generated by the general composite explicit scheme (3.3).

Theorem 3.2 Let $\left\{x_{n}\right\}$ be the sequence generated by the explicit scheme (3.3), where $\left\{\alpha_{n}\right\}$ and $\left\{\beta_{n}\right\}$ satisfy the following condition:

(C1) $\left\{\alpha_{n}\right\} \subset[0,1]$ and $\left\{\beta_{n}\right\} \subset(0,1], \alpha_{n} \rightarrow 0$ and $\beta_{n} \rightarrow 0$ as $n \rightarrow \infty$.

Let LIM be a Banach limit. Then

$$
\operatorname{LIM}_{n}\left(\left\langle(A-I) \tilde{x}, \tilde{x}-x_{n}\right\rangle\right) \leq 0,
$$

where $\widetilde{x}=\lim _{t \rightarrow 0^{+}} x_{t}$ with $x_{t}$ being defined by

$$
x_{t}=\left(I-\theta_{t} A\right) S x_{t}+\theta_{t}\left[t \gamma V x_{t}+(I-t \mu F) S x_{t}\right] \text {, }
$$

where $S: H \rightarrow H$ is defined by $S x=\lambda x+(1-\lambda)$ Tx for $0 \leq k \leq \lambda<1$.

Proof First, note that from the condition (C1), without loss of generality, we assume that $0<\beta_{n} \leq\|A\|^{-1}$ for all $n \geq 0$.

Let $\left\{x_{t}\right\}$ be the net generated by (3.8). Since $S$ is a nonexpansive mapping on $H$, by Theorem 3.1 with $T_{t}=S$ and Lemma 2.5, there exists $\lim _{t \rightarrow 0} x_{t} \in \operatorname{Fix}(S)=\operatorname{Fix}(T)$. Denote it by $\tilde{x}$. Moreover, $\tilde{x}$ is the unique solution of the variational inequality (3.2). From Proposition 3.1(i) with $T_{t}=S$, we know that $\left\{x_{t}\right\}$ is bounded, so are $\left\{V x_{t}\right\}$ and $\left\{F S x_{t}\right\}$.

First of all, let us show that $\left\{x_{n}\right\}$ is bounded. To this end, take $p \in \operatorname{Fix}(T)=\operatorname{Fix}\left(T_{n}\right)$, Then it follows that

$$
\begin{aligned}
\left\|y_{n}-p\right\| & =\left\|\alpha_{n} \gamma V x_{n}+\left(I-\alpha_{n} \mu F\right) T_{n} x_{n}-p\right\| \\
& =\left\|\alpha_{n}\left(\gamma V x_{n}-\mu F p\right)+\left(I-\alpha_{n} \mu F\right) T_{n} x_{n}-\left(I-\alpha_{n} \mu F\right) T_{n} p\right\| \\
& \leq\left(1-\alpha_{n}(\tau-\gamma l)\right)\left\|x_{n}-p\right\|+\alpha_{n}\|\gamma V p-\mu F p\|,
\end{aligned}
$$

and hence

$$
\begin{aligned}
\left\|x_{n+1}-p\right\| & =\left\|\left(I-\beta_{n} A\right) T_{n} x_{n}+\beta_{n} y_{n}-p\right\| \\
& =\left\|\left(I-\beta_{n} A\right) T_{n} x_{n}-\left(I-\beta_{n} A\right) T_{n} p+\beta_{n}\left(y_{n}-p\right)+\beta_{n}(I-A) p\right\|
\end{aligned}
$$




$$
\begin{aligned}
\leq & \left\|\left(I-\beta_{n} A\right) T_{n} x_{n}-\left(I-\beta_{n} A\right) T_{n} p\right\|+\beta_{n}\left\|y_{n}-p\right\|+\beta_{n}\|I-A\|\|p\| \\
\leq & \left(1-\beta_{n} \bar{\gamma}\right)\left\|x_{n}-p\right\|+\beta_{n}\left[\left(1-\alpha_{n}(\tau-\gamma l)\right)\left\|x_{n}-p\right\|+\alpha_{n}\|\gamma V p-\mu F p\|\right] \\
& +\beta_{n}\|I-A\|\|p\| \\
\leq & \left(1-\beta_{n}(\bar{\gamma}-1)\right)\left\|x_{n}-p\right\|+\beta_{n}(\|\gamma V p-\mu F p\|+\|I-A\|\|p\|) \\
= & \left(1-\beta_{n}(\bar{\gamma}-1)\right)\left\|x_{n}-p\right\|+\beta_{n}(\bar{\gamma}-1) \frac{\|\gamma V p-\mu F p\|+\|I-A\|\|p\|}{\bar{\gamma}-1} \\
\leq & \max \left\{\left\|x_{n}-p\right\|, \frac{\|\gamma V p-\mu F p\|+\|I-A\|\|p\|}{\bar{\gamma}-1}\right\} .
\end{aligned}
$$

By induction

$$
\left\|x_{n}-p\right\| \leq \max \left\{\left\|x_{0}-p\right\|, \frac{\|\gamma V p-\mu F p\|+\|I-A\|\|p\|}{\bar{\gamma}-1}\right\}, \quad \forall n \geq 0 .
$$

This implies that $\left\{x_{n}\right\}$ is bounded and so are $\left\{T x_{n}\right\},\left\{T_{n} x_{n}\right\},\left\{F T_{n} x_{n}\right\},\left\{V x_{n}\right\}$, and $\left\{y_{n}\right\}$. As a consequence, with the control condition (C1), we get

$$
\left\|x_{n+1}-T_{n} x_{n}\right\|=\beta_{n}\left\|y_{n}-A T_{n} x_{n}\right\| \rightarrow 0 \quad(n \rightarrow \infty),
$$

and

$$
\begin{aligned}
\left\|S x_{t}-x_{n+1}\right\| & \leq\left\|S x_{t}-S x_{n}\right\|+\left\|S x_{n}-T_{n} x_{n}\right\|+\left\|T_{n} x_{n}-x_{n+1}\right\| \\
& \leq\left\|x_{t}-x_{n}\right\|+\left|\lambda-\lambda_{n}\right|\left\|x_{n}-T x_{n}\right\|+\left\|T_{n} x_{n}-x_{n+1}\right\| \\
& =\left\|x_{t}-x_{n}\right\|+e_{n},
\end{aligned}
$$

where $e_{n}=\left|\lambda-\lambda_{n}\right|\left\|x_{n}-T x_{n}\right\|+\left\|x_{n+1}-T_{n} x_{n}\right\| \rightarrow 0$ as $n \rightarrow \infty$. Also observing that $A$ is strongly positive, we have

$$
\begin{aligned}
\left\langle A x_{t}-A x_{n}, x_{t}-x_{n}\right\rangle & =\left\langle A\left(x_{t}-x_{n}\right), x_{t}-x_{n}\right\rangle \\
& \geq \bar{\gamma}\left\|x_{t}-x_{n}\right\|^{2} .
\end{aligned}
$$

Now, by (3.8), we have

$$
\begin{aligned}
x_{t}-x_{n+1} & =\left(I-\theta_{t} A\right) S x_{t}+\theta_{t}\left[t \gamma V x_{t}+(I-t \mu F) S x_{t}\right]-x_{n+1} \\
& =\left(I-\theta_{n} A\right) S x_{t}-\left(I-\theta_{t} A\right) x_{n+1}+\theta_{t}\left[t \gamma V x_{t}+(I-t \mu F) S x_{t}-A x_{n+1}\right] .
\end{aligned}
$$

Applying Lemma 2.1, we have

$$
\begin{aligned}
\left\|x_{t}-x_{n+1}\right\|^{2} \leq & \left\|\left(I-\theta_{t} A\right) S x_{t}-\left(I-\theta_{t} A\right) x_{n+1}\right\|^{2} \\
& +2 \theta_{t}\left\langle S x_{t}-t\left(\mu F S x_{t}-\gamma V x_{t}\right)-A x_{n+1}, x_{t}-x_{n+1}\right\rangle \\
\leq & \left(1-\theta_{t} \bar{\gamma}\right)^{2}\left\|S x_{t}-x_{n+1}\right\|^{2}+2 \theta_{t}\left\langle S x_{t}-x_{t}, x_{t}-x_{n+1}\right\rangle \\
& -2 \theta_{t} t\left\langle\mu F S x_{t}-\gamma V x_{t}, x_{t}-x_{n+1}\right\rangle+2 \theta_{t}\left\langle x_{t}-A x_{n+1}, x_{t}-x_{n+1}\right\rangle .
\end{aligned}
$$


Using (3.9) and (3.10) in (3.11), we obtain

$$
\begin{aligned}
& \left\|x_{t}-x_{n+1}\right\|^{2} \\
& \leq\left(1-\theta_{t} \bar{\gamma}\right)^{2}\left\|S x_{t}-x_{n+1}\right\|^{2}+2 \theta_{t}\left\langle S x_{t}-x_{t}, x_{t}-x_{n+1}\right\rangle \\
& +2 \theta_{t} t\left\langle\gamma V x_{t}-\mu F S x_{t}, x_{t}-x_{n+1}\right\rangle \\
& +2 \theta_{t}\left\langle x_{t}-A x_{n+1}, x_{t}-x_{n+1}\right\rangle \\
& \leq\left(1-\theta_{t} \bar{\gamma}\right)^{2}\left(\left\|x_{t}-x_{n}\right\|+e_{n}\right)^{2}+2 \theta_{t}\left\|S x_{t}-x_{t}\right\|\left\|x_{t}-x_{n+1}\right\| \\
& +2 \theta_{t} t\left\|\gamma V x_{t}-\mu F S x_{t}\right\|\left\|x_{t}-x_{n+1}\right\|+2 \theta_{t}\left\langle x_{t}-A x_{n+1}, x_{t}-x_{n+1}\right\rangle \\
& =\left(\theta_{t}^{2} \bar{\gamma}-2 \theta_{t}\right) \bar{\gamma}\left\|x_{t}-x_{n}\right\|^{2}+\left\|x_{t}-x_{n}\right\|^{2}+\left(1-\theta_{t} \bar{\gamma}\right)^{2}\left(2\left\|x_{t}-x_{n}\right\| e_{n}+e_{n}^{2}\right) \\
& +2 \theta_{t}\left\|S x_{t}-x_{t}\right\|\left\|x_{t}-x_{n+1}\right\|+2 \theta_{t} t\left\|\gamma V x_{t}-\mu F S x_{t}\right\|\left\|x_{t}-x_{n+1}\right\| \\
& +2 \theta_{t}\left\langle x_{t}-A x_{n+1}, x_{t}-x_{n+1}\right\rangle \\
& \leq\left(\theta_{t}^{2} \bar{\gamma}-2 \theta_{t}\right)\left\langle A x_{t}-A x_{n}, x_{t}-x_{n}\right\rangle+\left\|x_{t}-x_{n}\right\|^{2}+\left(1-\theta_{t} \bar{\gamma}\right)^{2}\left(2\left\|x_{t}-x_{n}\right\| e_{n}+e_{n}^{2}\right) \\
& +2 \theta_{t}\left\|S x_{t}-x_{t}\right\|\left\|x_{t}-x_{n+1}\right\|+2 \theta_{t} t\left\|\gamma V x_{t}-\mu F S x_{t}\right\|\left\|x_{t}-x_{n+1}\right\| \\
& +2 \theta_{t}\left\langle x_{t}-A x_{n+1}, x_{t}-x_{n+1}\right\rangle \\
& =\theta_{t}^{2} \bar{\gamma}\left\langle A x_{t}-A x_{n}, x_{t}-x_{n}\right\rangle+\left\|x_{t}-x_{n}\right\|^{2}+\left(1-\theta_{t} \bar{\gamma}\right)^{2}\left(2\left\|x_{t}-x_{n}\right\| e_{n}+e_{n}^{2}\right) \\
& +2 \theta_{t}\left\|S x_{t}-x_{t}\right\|\left\|x_{t}-x_{n+1}\right\|+2 \theta_{t} t\left\|\gamma V x_{t}-\mu F\left(S x_{t}\right)\right\|\left\|x_{t}-x_{n+1}\right\| \\
& +2 \theta_{t}\left[\left\langle x_{t}-A x_{n+1}, x_{t}-x_{n+1}\right\rangle-\left\langle A x_{t}-A x_{n}, x_{t}-x_{n}\right\rangle\right] \\
& =\theta_{t}^{2} \bar{\gamma}\left\langle A\left(x_{t}-x_{n}\right), x_{t}-x_{n}\right\rangle+\left\|x_{t}-x_{n}\right\|^{2}+\left(1-\theta_{t} \bar{\gamma}\right)^{2}\left(2\left\|x_{t}-x_{n}\right\| e_{n}+e_{n}^{2}\right) \\
& +2 \theta_{t}\left\|S x_{t}-x_{t}\right\|\left\|x_{t}-x_{n+1}\right\|+2 \theta_{t} t\left\|\gamma V x_{t}-\mu F S x_{t}\right\|\left\|x_{t}-x_{n+1}\right\| \\
& +2 \theta_{t}\left[\left\langle(I-A) x_{t}, x_{t}-x_{n+1}\right\rangle+\left\langle A\left(x_{t}-x_{n+1}\right), x_{t}-x_{n+1}\right\rangle\right. \\
& \left.-\left\langle A\left(x_{t}-x_{n}\right), x_{t}-x_{n}\right\rangle\right] \text {. }
\end{aligned}
$$

Applying the Banach limit LIM to (3.12), together with $\lim _{n \rightarrow \infty} e_{n}=0$, we have

$$
\begin{aligned}
\operatorname{LIM}_{n} & \left(\left\|x_{t}-x_{n+1}\right\|^{2}\right) \\
\leq & \theta_{t}^{2} \bar{\gamma} \operatorname{LIM}_{n}\left(\left\langle A\left(x_{t}-x_{n}\right), x_{t}-x_{n}\right\rangle\right)+\operatorname{LIM}_{n}\left(\left\|x_{t}-x_{n}\right\|^{2}\right) \\
& +2 \theta_{t}\left\|S x_{t}-x_{t}\right\| \operatorname{LIM}_{n}\left(\left\|x_{t}-x_{n+1}\right\|\right) \\
& +2 \theta_{t} t\left\|\gamma V x_{t}-\mu F S x_{t}\right\| \operatorname{LIM}_{n}\left(\left\|x_{t}-x_{n+1}\right\|\right) \\
& +2 \theta_{t}\left[\operatorname{LIM}_{n}\left(\left\langle(I-A) x_{t}, x_{t}-x_{n+1}\right\rangle\right)+\operatorname{LIM}_{n}\left(\left\langle A\left(x_{t}-x_{n+1}\right), x_{t}-x_{n+1}\right\rangle\right)\right. \\
& \left.-\operatorname{LIM}_{n}\left(\left\langle A\left(x_{t}-x_{n}\right), x_{t}-x_{n}\right\rangle\right)\right] .
\end{aligned}
$$

Using the property $\operatorname{LIM}_{n}\left(a_{n}\right)=\operatorname{LIM}_{n}\left(a_{n+1}\right)$ of the Banach limit in (3.13), we obtain

$$
\begin{aligned}
& \operatorname{LIM}_{n}\left(\left\langle(A-I) x_{t}, x_{t}-x_{n}\right\rangle\right) \\
& \quad=\operatorname{LIM}_{n}\left(\left\langle(A-I) x_{t}, x_{t}-x_{n+1}\right\rangle\right) \\
& \quad \leq \frac{\theta_{t} \bar{\gamma}}{2} \operatorname{LIM}_{n}\left(\left\langle A\left(x_{t}-x_{n}\right), x_{t}-x_{n}\right\rangle\right)
\end{aligned}
$$




$$
\begin{aligned}
& +\frac{1}{2 \theta_{t}}\left[\operatorname{LIM}_{n}\left(\left\|x_{t}-x_{n}\right\|^{2}\right)-\operatorname{LIM}_{n}\left(\left\|x_{t}-x_{n+1}\right\|^{2}\right)\right] \\
& +\left\|S x_{t}-x_{t}\right\| \operatorname{LIM}_{n}\left(\left\|x_{t}-x_{n}\right\|\right)+t\left\|\gamma V x_{t}-\mu F S x_{t}\right\| \operatorname{LIM}_{n}\left(\left\|x_{t}-x_{n}\right\|\right) \\
& +\operatorname{LIM}_{n}\left(\left\langle A\left(x_{t}-x_{n+1}\right), x_{t}-x_{n+1}\right\rangle\right)-\operatorname{LIM}_{n}\left(\left\langle A\left(x_{t}-x_{n}\right), x_{t}-x_{n}\right\rangle\right) \\
= & \frac{\theta_{t} \bar{\gamma}}{2} \operatorname{LIM}_{n}\left(\left\langle A\left(x_{t}-x_{n}\right), x_{t}-x_{n}\right\rangle\right) \\
& +\left\|S x_{t}-x_{t}\right\| \operatorname{LIM}_{n}\left(\left\|x_{t}-x_{n}\right\|\right)+t\left\|\gamma V x_{t}-\mu F S x_{t}\right\| \operatorname{LIM}_{n}\left(\left\|x_{t}-x_{n}\right\|\right) .
\end{aligned}
$$

Since

$$
\begin{aligned}
\theta_{t}\left|A\left(x_{t}-x_{n}\right), x_{t}-x_{n}\right\rangle & \leq \theta_{t}\|A\|\left\|x_{t}-x_{n}\right\|^{2} \\
& \leq \theta_{t} K \rightarrow 0 \quad(\text { as } t \rightarrow 0)
\end{aligned}
$$

where $\|A\|\left\|x_{t}-x_{n}\right\|^{2} \leq K$,

$$
\left\|S x_{t}-x_{t}\right\| \rightarrow 0, \quad \text { and } \quad t\left\|\gamma V x_{t}-\mu F S x_{t}\right\| \rightarrow 0 \quad(\text { as } t \rightarrow 0) \text {, }
$$

we conclude from (3.14)-(3.16) that

$$
\begin{aligned}
\operatorname{LIM}_{n}\left(\left\langle(A-I) \tilde{x}, \tilde{x}-x_{n}\right\rangle\right) \leq & \limsup _{t \rightarrow 0} \operatorname{LIM}_{n}\left(\left\langle(A-I) x_{t}, x_{t}-x_{n}\right\rangle\right) \\
\leq & \limsup _{t \rightarrow 0} \frac{\theta_{t} \bar{\gamma}}{2} \operatorname{LIM}_{n}\left(\left\langle A\left(x_{t}-x_{n}\right), x_{t}-x_{n}\right\rangle\right) \\
& +\limsup _{t \rightarrow 0}\left\|S x_{t}-x_{t}\right\| \operatorname{LIM}_{n}\left(\left\|x_{t}-x_{n}\right\|\right) \\
& +\limsup _{t \rightarrow 0} t\left\|\gamma V x_{t}-\mu F S x_{t}\right\| \operatorname{LIM}_{n}\left(\left\|x_{t}-x_{n}\right\|\right) \\
= & 0 .
\end{aligned}
$$

This completes the proof.

Now, using Theorem 3.2, we establish strong convergence of the sequence $\left\{x_{n}\right\}$ generated by the general composite explicit scheme (3.3) to a fixed point $\tilde{x}$ of $T$, which is also the unique solution of the variational inequality (3.2).

Theorem 3.3 Let $\left\{x_{n}\right\}$ be the sequence generated by the explicit scheme (3.3), where $\left\{\alpha_{n}\right\}$ and $\left\{\beta_{n}\right\}$ satisfy the following conditions:

(C1) $\left\{\alpha_{n}\right\} \subset[0,1]$ and $\left\{\beta_{n}\right\} \subset(0,1], \alpha_{n} \rightarrow 0$ and $\beta_{n} \rightarrow 0$ as $n \rightarrow \infty$;

(C2) $\sum_{n=0}^{\infty} \beta_{n}=\infty$.

If $\left\{x_{n}\right\}$ is weakly asymptotically regular, then $\left\{x_{n}\right\}$ converges strongly to $\tilde{x} \in \operatorname{Fix}(T)$, which is the unique solution of the variational inequality (3.2).

Proof First, note that from the condition (C1), without loss of generality, we assume that $\alpha_{n} \tau<1$ and $\frac{2 \beta_{n}(\bar{\gamma}-1)}{1-\beta_{n}}<1$ for all $n \geq 0$.

Let $x_{t}$ be defined by (3.8), that is,

$$
x_{t}=\left(I-\theta_{t} A\right) S x_{t}+\theta_{t}\left[S x_{t}-t\left(\mu F\left(S x_{t}\right)-\gamma V x_{t}\right)\right]
$$


for $t \in\left(0, \min \left\{1, \frac{2-\bar{\gamma}}{\tau-\gamma l}\right\}\right)$, where $S x=\lambda x+(1-\lambda) T x$ for $0 \leq k \leq \lambda<1$, and $\lim _{t \rightarrow 0} x_{t}:=\tilde{x} \in$ $\operatorname{Fix}(S)=\operatorname{Fix}(T)$ (by using Theorem 3.1 and Lemma 2.5(iii)). Then $\widetilde{x}$ is the unique solution of the variational inequality (3.2).

We divide the proof into several steps as follows.

Step 1. We see that

$$
\left\|x_{n}-p\right\| \leq \max \left\{\left\|x_{0}-p\right\|, \frac{\|\gamma V p-\mu F p\|+\|I-A\|\|p\|}{\bar{\gamma}-1}\right\}, \quad \forall n \geq 0,
$$

for all $p \in \operatorname{Fix}(T)$ as in the proof of Theorem 3.2. Hence $\left\{x_{n}\right\}$ is bounded and so are $\left\{T x_{n}\right\}$, $\left\{T_{n} x_{n}\right\},\left\{F T_{n} x_{n}\right\},\left\{V x_{n}\right\}$, and $\left\{y_{n}\right\}$.

Step 2. We show that $\lim _{\sup _{n \rightarrow \infty}}\left\langle(I-A) \widetilde{x}, x_{n}-\widetilde{x}\right\rangle \leq 0$. To this end, put

$$
a_{n}:=\left\langle(A-I) \widetilde{x}, \widetilde{x}-x_{n}\right\rangle, \quad \forall n \geq 0 .
$$

Then Theorem 3.2 implies that $\operatorname{LIM}_{n}\left(a_{n}\right) \leq 0$ for any Banach limit LIM. Since $\left\{x_{n}\right\}$ is bounded, there exists a subsequence $\left\{x_{n_{j}}\right\}$ of $\left\{x_{n}\right\}$ such that

$$
\limsup _{n \rightarrow \infty}\left(a_{n+1}-a_{n}\right)=\lim _{j \rightarrow \infty}\left(a_{n_{j}+1}-a_{n_{j}}\right)
$$

and $x_{n_{j}} \rightarrow v \in H$. This implies that $x_{n_{j+1}} \rightarrow v$ since $\left\{x_{n}\right\}$ is weakly asymptotically regular. Therefore, we have

$$
w-\lim _{j \rightarrow \infty}\left(\widetilde{x}-x_{n_{j}+1}\right)=w-\lim _{j \rightarrow \infty}\left(\widetilde{x}-x_{n_{j}}\right)=(\widetilde{x}-v),
$$

and so

$$
\limsup _{n \rightarrow \infty}\left(a_{n+1}-a_{n}\right)=\lim _{j \rightarrow \infty}\left\langle(A-I) \widetilde{x},\left(\widetilde{x}-x_{n_{j}+1}\right)-\left(\widetilde{x}-x_{n_{j}}\right)\right\rangle=0 .
$$

Then Lemma 2.2 implies that $\lim \sup _{n \rightarrow \infty} a_{n} \leq 0$, that is,

$$
\limsup _{n \rightarrow \infty}\left\langle(I-A) \tilde{x}, x_{n}-\tilde{x}\right\rangle=\limsup _{n \rightarrow \infty}\left\langle(A-I) \tilde{x}, \tilde{x}-x_{n}\right\rangle \leq 0 .
$$

Step 3. We show that $\lim _{n \rightarrow \infty}\left\|x_{n}-\tilde{x}\right\|=0$. By using (3.3) and $T_{n} \tilde{x}=\tilde{x}$, we have

$$
y_{n}-\tilde{x}=\left(I-\alpha_{n} \mu F\right) T_{n} x_{n}-\left(I-\alpha_{n} \mu F\right) T_{n} \tilde{x}+\alpha_{n}\left(\gamma V x_{n}-\mu F \widetilde{x}\right),
$$

and

$$
x_{n+1}-\tilde{x}=\left(I-\beta_{n} A\right)\left(T_{n} x_{n}-T_{n} \widetilde{x}\right)+\beta_{n}\left(y_{n}-\widetilde{x}\right)+\beta_{n}(I-A) \widetilde{x} .
$$

Applying Lemma 2.1, Lemma 2.6 and Lemma 2.7, we obtain

$$
\begin{aligned}
\left\|y_{n}-\widetilde{x}\right\|^{2} & =\left\|\left(I-\mu \alpha_{n} F\right) T_{n} x_{n}-\left(I-\mu \alpha_{n} F\right) T_{n} \widetilde{x}+\alpha_{n}\left(\gamma V x_{n}-\mu F \widetilde{x}\right)\right\|^{2} \\
& \leq\left\|\left(I-\mu \alpha_{n} F\right) T_{n} x_{n}-\left(I-\mu \alpha_{n} F\right) T_{n} \widetilde{x}\right\|^{2}+2 \alpha_{n}\left\langle\gamma V x_{n}-\mu F \widetilde{x}, y_{n}-\widetilde{x}\right\rangle
\end{aligned}
$$




$$
\begin{aligned}
& \leq\left(1-\alpha_{n} \tau\right)^{2}\left\|x_{n}-\tilde{x}\right\|^{2}+2 \alpha_{n}\left\|\gamma V x_{n}-\mu F \tilde{x}\right\|\left\|y_{n}-\tilde{x}\right\| \\
& \leq\left\|x_{n}-\tilde{x}\right\|^{2}+2 \alpha_{n}\left\|\gamma V x_{n}-\mu F \tilde{x}\right\|\left\|y_{n}-\tilde{x}\right\|
\end{aligned}
$$

and hence

$$
\begin{aligned}
\left\|x_{n+1}-\tilde{x}\right\|^{2}= & \left\|\left(I-\beta_{n} A\right)\left(T_{n} x_{n}-T_{n} \tilde{x}\right)+\beta_{n}\left(y_{n}-\tilde{x}\right)+\beta_{n}(I-A) \widetilde{x}\right\|^{2} \\
\leq & \left\|\left(I-\beta_{n} A\right)\left(T_{n} x_{n}-T_{n} \tilde{x}\right)\right\|^{2}+2 \beta_{n}\left\langle y_{n}-\tilde{x}, x_{n+1}-\tilde{x}\right\rangle \\
& +2 \beta_{n}\left\langle(I-A) \tilde{x}, x_{n+1}-\tilde{x}\right\rangle \\
\leq & \left(1-\beta_{n} \bar{\gamma}\right)^{2}\left\|x_{n}-\tilde{x}\right\|^{2}+2 \beta_{n}\left\|y_{n}-\tilde{x}\right\|\left\|x_{n+1}-\tilde{x}\right\| \\
& +2 \beta_{n}\left\langle(I-A) \widetilde{x}, x_{n+1}-\tilde{x}\right\rangle \\
\leq & \left(1-\beta_{n} \bar{\gamma}\right)^{2}\left\|x_{n}-\tilde{x}\right\|^{2}+\beta_{n}\left(\left\|y_{n}-\tilde{x}\right\|^{2}+\left\|x_{n+1}-\tilde{x}\right\|^{2}\right) \\
& +2 \beta_{n}\left\langle(I-A) \tilde{x}, x_{n+1}-\tilde{x}\right\rangle \\
\leq & \left(1-\beta_{n} \bar{\gamma}\right)^{2}\left\|x_{n}-\tilde{x}\right\|^{2} \\
& +\beta_{n}\left[\left\|x_{n}-\tilde{x}\right\|^{2}+2 \alpha_{n}\left\|\gamma V x_{n}-\mu F \tilde{x}\right\|\left\|y_{n}-\tilde{x}\right\|\right] \\
& +\beta_{n}\left\|x_{n+1}-\tilde{x}\right\|^{2}+2 \beta_{n}\left\langle(I-A) \widetilde{x}, x_{n+1}-\tilde{x}\right\rangle \\
= & {\left[\left(1-\beta_{n} \bar{\gamma}\right)^{2}+\beta_{n}\right]\left\|x_{n}-\tilde{x}\right\|^{2}+2 \alpha_{n} \beta_{n}\left\|\gamma V x_{n}-\mu F \widetilde{x}\right\|\left\|y_{n}-\tilde{x}\right\| }
\end{aligned}
$$

It then follows from (3.17) that

$$
\begin{aligned}
\left\|x_{n+1}-\tilde{x}\right\|^{2} \leq & \frac{\left(1-\beta_{n} \bar{\gamma}\right)^{2}+\beta_{n}}{1-\beta_{n}}\left\|x_{n}-\tilde{x}\right\|^{2} \\
& +\frac{\beta_{n}}{1-\beta_{n}}\left[2 \alpha_{n}\left\|\gamma V x_{n}-\mu F \tilde{x}\right\|\left\|y_{n}-\tilde{x}\right\|+2\left\langle(I-A) \tilde{x}, x_{n+1}-\tilde{x}\right\rangle\right] \\
= & \left(1-\frac{2 \beta_{n}(\bar{\gamma}-1)}{1-\beta_{n}}\right)\left\|x_{n}-\tilde{x}\right\|^{2} \\
& +\frac{2 \beta_{n}(\bar{\gamma}-1)}{1-\beta_{n}} \cdot \frac{1}{2(\bar{\gamma}-1)}\left[2 \alpha_{n}\left\|\gamma V x_{n}-\mu F \tilde{x}\right\|\left\|y_{n}-\tilde{x}\right\|\right. \\
& \left.+\beta_{n} \bar{\gamma}^{2}\left\|x_{n}-\tilde{x}\right\|^{2}+2\left\langle(I-A) \widetilde{x}, x_{n+1}-\tilde{x}\right\rangle\right] \\
= & \left(1-\omega_{n}\right)\left\|x_{n}-\tilde{x}\right\|^{2}+\omega_{n} \delta_{n},
\end{aligned}
$$

where

$$
\begin{aligned}
& \omega_{n}=\frac{2 \beta_{n}(\bar{\gamma}-1)}{1-\beta_{n}} \text { and } \\
& \delta_{n}=\frac{1}{2(\bar{\gamma}-1)}\left[2 \alpha_{n}\left\|\gamma V x_{n}-\mu F \tilde{x}\right\|\left\|y_{n}-\tilde{x}\right\|+\beta_{n} \bar{\gamma}^{2}\left\|x_{n}-\tilde{x}\right\|^{2}+2\left\langle(I-A) \tilde{x}, x_{n+1}-\tilde{x}\right\rangle\right] .
\end{aligned}
$$

It can easily be seen from Step 2 and conditions $(\mathrm{C} 1)$ and (C2) that $\omega_{n} \rightarrow 0, \sum_{n=0}^{\infty} \omega_{n}=$ $\infty$ and $\lim \sup _{n \rightarrow \infty} \delta_{n} \leq 0$. From Lemma 2.3 with $r_{n}=0$, we conclude that $\lim _{n \rightarrow \infty} \| x_{n}-$ $\tilde{x} \|=0$. This completes the proof. 
Corollary 3.2 Let $\left\{x_{n}\right\}$ be the sequence generated by the explicit scheme (3.3). Assume that the sequence $\left\{\alpha_{n}\right\}$ and $\left\{\beta_{n}\right\}$ satisfy the conditions (C1) and (C2) in Theorem 3.3. If $\left\{x_{n}\right\}$ is asymptotically regular, then $\left\{x_{n}\right\}$ converges strongly to $\tilde{x} \in \operatorname{Fix}(T)$, which is the unique solution of the variational inequality (3.2).

Putting $\mu=1, F=I$ and $\gamma=1$ in Theorem 3.3, we obtain the following.

Corollary 3.3 Let $\left\{x_{n}\right\}$ be generated by the following iterative scheme:

$$
\left\{\begin{array}{l}
y_{n}=\alpha_{n} V x_{n}+\left(1-\alpha_{n}\right) T_{n} x_{n}, \\
x_{n+1}=\left(I-\beta_{n} A\right) T_{n} x_{n}+\beta_{n} y_{n}, \quad \forall n \geq 0 .
\end{array}\right.
$$

Assume that the sequence $\left\{\alpha_{n}\right\}$ and $\left\{\beta_{n}\right\}$ satisfy the conditions $(\mathrm{C} 1)$ and $(\mathrm{C} 2)$ in Theorem 3.3. If $\left\{x_{n}\right\}$ is weakly asymptotically regular, then $\left\{x_{n}\right\}$ converges strongly to $\tilde{x} \in \operatorname{Fix}(T)$, which is the unique solution of the variational inequality (3.2).

Putting $\alpha_{n}=0, \forall n \geq 0$ in Corollary 3.3, we get the following.

Corollary 3.4 Let $\left\{x_{n}\right\}$ be generated by the following iterative scheme:

$$
x_{n+1}=\left(I-\beta_{n} A\right) T_{n} x_{n}+\beta_{n} T_{n} x_{n}, \quad \forall n \geq 0 .
$$

Assume that the sequence $\left\{\beta_{n}\right\}$ satisfies the conditions $(\mathrm{C} 1)$ and $(\mathrm{C} 2)$ in Theorem 3.3 with $\alpha_{n}=0, \forall n \geq 0$. If $\left\{x_{n}\right\}$ is weakly asymptotically regular, then $\left\{x_{n}\right\}$ converges strongly to $\tilde{x} \in \operatorname{Fix}(T)$, which is the unique solution of the variational inequality (3.2).

Remark 3.1 If $\left\{\alpha_{n}\right\},\left\{\beta_{n}\right\}$ in Corollary 3.2 and $\left\{\lambda_{n}\right\}$ in $T_{n}$ satisfy conditions (C2) and

(C3) $\sum_{n=0}^{\infty}\left|\alpha_{n+1}-\alpha_{n}\right|<\infty$ and $\sum_{n=0}^{\infty}\left|\beta_{n+1}-\beta_{n}\right|<\infty$; or

(C4) $\sum_{n=0}^{\infty}\left|\alpha_{n+1}-\alpha_{n}\right|<\infty$ and $\lim _{n \rightarrow \infty} \frac{\beta_{n}}{\beta_{n+1}}=1$ or, equivalently, $\lim _{n \rightarrow \infty} \frac{\alpha_{n}-\alpha_{n+1}}{\alpha_{n+1}}=0$ and $\lim _{n \rightarrow \infty} \frac{\beta_{n}-\beta_{n+1}}{\beta_{n+1}}=0$; or,

(C5) $\sum_{n=0}^{\infty}\left|\alpha_{n+1}-\alpha_{n}\right|<\infty$ and $\left|\beta_{n+1}-\beta_{n}\right| \leq o\left(\beta_{n+1}\right)+\sigma_{n}, \sum_{n=0}^{\infty} \sigma_{n}<\infty$ (the perturbed control condition);

(C6) $\sum_{n=0}\left|\lambda_{n+1}-\lambda_{n}\right|<\infty$,

then the sequence $\left\{x_{n}\right\}$ generated by (3.3) is asymptotically regular. Now we give only the proof in the case when $\left\{\alpha_{n}\right\},\left\{\beta_{n}\right\}$, and $\left\{\lambda_{n}\right\}$ satisfy the conditions (C2), (C5), and (C6). By Step 1 in the proof of Theorem 3.3, there exists a constant $M>0$ such that, for all $n \geq 0$,

$$
\left\|x_{n}-T x_{n}\right\| \leq M, \quad \mu\left\|F T_{n} x_{n}\right\|+\gamma\left\|V x_{n}\right\| \leq M, \quad \text { and } \quad\|A\|\left\|T_{n} x_{n}\right\|+\left\|y_{n}\right\| \leq M .
$$

Next, we notice that

$$
\begin{aligned}
\left\|T_{n} x_{n}-T_{n-1} x_{n-1}\right\| & \leq\left\|T_{n} x_{n}-T_{n} x_{n-1}\right\|+\left\|T_{n} x_{n-1}-T_{n-1} x_{n-1}\right\| \\
& \leq\left\|x_{n}-x_{n-1}\right\|+\left|\lambda_{n}-\lambda_{n-1}\right|\left\|x_{n-1}-T x_{n-1}\right\| \\
& \leq\left\|x_{n}-x_{n-1}\right\|+\left|\lambda_{n}-\lambda_{n-1}\right| M .
\end{aligned}
$$


So we obtain, for all $n \geq 0$,

$$
\begin{aligned}
\| y_{n}- & y_{n-1} \| \\
= & \| \alpha_{n} \gamma\left(V x_{n}-V x_{n-1}\right)+\gamma\left(\alpha_{n}-\alpha_{n-1}\right) V x_{n-1} \\
& \quad+\left(I-\alpha_{n} \mu F\right) T_{n} x_{n}-\left(I-\alpha_{n} \mu F\right) T_{n-1} x_{n-1}+\mu\left(\alpha_{n}-\alpha_{n-1}\right) F T_{n-1} x_{n-1} \| \\
\leq & \left(1-\alpha_{n}(\tau-\gamma l)\right)\left\|T_{n} x_{n}-T_{n-1} x_{n-1}\right\|+\left|\alpha_{n}-\alpha_{n-1}\right|\left(\gamma\left\|V x_{n-1}\right\|+\mu\left\|F T_{n-1} x_{n-1}\right\|\right) \\
\leq & \left(1-\alpha_{n}(\tau-\gamma l)\right)\left(\left\|x_{n}-x_{n-1}\right\|+\left|\lambda_{n}-\lambda_{n-1}\right| M\right)+\left|\alpha_{n}-\alpha_{n-1}\right| M,
\end{aligned}
$$

and hence

$$
\begin{aligned}
\| x_{n+1} & -x_{n} \| \\
= & \left\|\left(I-\beta_{n} A\right) T_{n} x_{n}+\beta_{n} y_{n}-\left(I-\beta_{n-1} A\right) T_{n-1} x_{n-1}-\beta_{n-1} y_{n-1}\right\| \\
\leq & \left\|\left(I-\beta_{n} A\right)\left(T_{n} x_{n}-T_{n-1} x_{n-1}\right)\right\| \\
& +\left|\beta_{n}-\beta_{n-1}\right|\|A\|\left\|T_{n-1} x_{n-1}\right\|+\beta_{n}\left\|y_{n}-y_{n-1}\right\|+\left|\beta_{n}-\beta_{n-1}\right|\left\|y_{n-1}\right\| \\
\leq & \left(1-\beta_{n} \bar{\gamma}\right)\left\|T_{n} x_{n}-T_{n-1} x_{n-1}\right\| \\
& +\beta_{n}\left(1-\alpha_{n}(\tau-\gamma l)\right)\left(\left\|x_{n}-x_{n-1}\right\|+\left|\lambda_{n}-\lambda_{n-1}\right| M\right)+\beta_{n}\left|\alpha_{n}-\alpha_{n-1}\right| M \\
& +\left|\beta_{n}-\beta_{n-1}\right|\left(\|A\|\left\|T_{n-1} x_{n-1}\right\|+\left\|y_{n-1}\right\|\right) \\
\leq & \left(1-\beta_{n} \bar{\gamma}\right)\left(\left\|x_{n}-x_{n-1}\right\|+\left|\lambda_{n}-\lambda_{n-1}\right| M\right) \\
& +\beta_{n}\left[\left(1-\alpha_{n}(\tau-\gamma l)\right)\left(\left\|x_{n}-x_{n-1}\right\|+\left|\lambda_{n}-\lambda_{n-1}\right| M\right)+\left|\alpha_{n}-\alpha_{n-1}\right| M\right] \\
& +\left|\beta_{n}-\beta_{n-1}\right| M \\
\leq & \left(1-\beta_{n} \bar{\gamma}\right)\left\|x_{n}-x_{n-1}\right\|+\left|\lambda_{n}-\lambda_{n-1}\right| M \\
& +\beta_{n}\left\|x_{n}-x_{n-1}\right\|+\left|\lambda_{n}-\lambda_{n-1}\right| M+\left|\alpha_{n}-\alpha_{n-1}\right| M+\left|\beta_{n}-\beta_{n-1}\right| M \\
= & \left(1-\beta_{n}(\bar{\gamma}-1)\right)\left\|x_{n}-x_{n-1}\right\|+\left|\beta_{n}-\beta_{n-1}\right| M+2\left|\lambda_{n}-\lambda_{n-1}\right| M+\left|\alpha_{n}-\alpha_{n-1}\right| M \\
\leq & \left(1-\beta_{n}(\bar{\gamma}-1)\right)\left\|x_{n}-x_{n-1}\right\|+\left(o\left(\beta_{n}\right)+\sigma_{n-1}\right) M+\left|\alpha_{n}-\alpha_{n-1}\right| M \\
& +2\left|\lambda_{n}-\lambda_{n-1}\right| M .
\end{aligned}
$$

By taking $s_{n+1}=\left\|x_{n+1}-x_{n}\right\|, \omega_{n}=\beta_{n}(\bar{\gamma}-1), \omega_{n} \delta_{n}=M o\left(\beta_{n}\right)$ and $r_{n}=\left(\left|\alpha_{n}-\alpha_{n-1}\right|+\sigma_{n-1}+\right.$ $\left.2\left|\lambda_{n}-\lambda_{n-1}\right|\right) M$, from (3.18) we have

$$
s_{n+1} \leq\left(1-\omega_{n}\right) s_{n}+\omega_{n} \delta_{n}+r_{n} .
$$

Hence, by the conditions (C2), (C5), (C6), and Lemma 2.3, we obtain

$$
\lim _{n \rightarrow \infty}\left\|x_{n+1}-x_{n}\right\|=0 .
$$

In view of this observation, we have the following.

Corollary 3.5 Let $\left\{x_{n}\right\}$ be the sequence generated by the explicit scheme (3.3), where the sequences $\left\{\alpha_{n}\right\},\left\{\beta_{n}\right\}$, and $\left\{\lambda_{n}\right\}$ satisfy the conditions (C1), (C2), (C5), and (C6) (or the conditions (C1), (C2), (C3) and (C6), or the conditions (C1), (C2), (C4), and (C6)). Then $\left\{x_{n}\right\}$ 
converges strongly to $\tilde{x} \in \operatorname{Fix}(T)$, which is the unique solution of the variational inequality (3.2).

Remark 3.2 (1) Our results improve and extend the corresponding results of Ceng et al. [18] in the following respects:

(a) The nonexpansive mapping $S: H \rightarrow H$ in [18] is extended to the case of a $k$-strictly pseudocontractive mapping $T: H \rightarrow H$.

(b) The contractive mapping $f$ in [18] with constant $\alpha \in(0,1)$ is extended to the case of a Lipschitzian mapping $V$ with constant $l \geq 0$.

(c) The range $0<\gamma \alpha<\tau=\mu\left(\eta-\frac{\mu \rho^{2}}{2}\right)$ in [18] is extended to the case of range $0<\gamma l<\tau=1-\sqrt{1-\mu\left(2 \eta-\mu \rho^{2}\right)}$. (For this fact, see Remark 3.1 of [17].)

(2) We point out that the condition (C3) $\sum_{n=0}^{\infty}\left|\alpha_{n+1}-\alpha_{n}\right|<\infty$ and $\sum_{n=0}^{\infty}\left|\beta_{n+1}-\beta_{n}\right|<\infty$ in $\left[18\right.$, Theorem 3.2] is relaxed to the case of the weak asymptotic regularity on $\left\{x_{n}\right\}$ in Theorem 3.3.

(3) The condition (C5) on $\left\{\beta_{n}\right\}$ in Corollary 3.5 is independent of condition (C3) or (C4) in Remark 3.1, which was imposed in Theorem 3.2 of Ceng et al. [18]. For this fact, see [24, 25].

(4) Our results also complement and develop the corresponding ones given by Cho et al. [5] and Jung [6-8] for the strictly pseudocontractive mapping as well as Yamada [12], Marino and $\mathrm{Xu}$ [13], Tian [15] and Ceng et al. [17] for the nonexpansive mapping.

(5) For several iterative schemes based on hybrid steepest-descent method for generalized mixed equilibrium problems, variational inequality problems, and fixed point problems for strictly pseudocontractive mappings, we can also refer to [26-32] and the references therein.

Competing interests

The author declares to have no competing interests.

\section{Acknowledgements}

The author would like to thank the anonymous referees for their careful reading and valuable comments along with providing some recent related papers, which improved the presentation of this manuscript.

This study was supported by research funds from Dong-A University.

Received: 9 May 2014 Accepted: 21 July 2014 Published: 18 Aug 2014

References

1. Browder, FE: Fixed point theorems for noncompact mappings. Proc. Natl. Acad. Sci. USA 53, 1272-1276 (1965)

2. Browder, FE: Convergence of approximants to fixed points of nonexpansive nonlinear mappings in Banach spaces. Arch. Ration. Mech. Anal. 24, 82-90 (1967)

3. Browder, FE, Petryshn, WV: Construction of fixed points of nonlinear mappings Hilbert space. J. Math. Anal. Appl. 20, 197-228 (1967)

4. Acedo, GL, Xu, HK: Iterative methods for strictly pseudo-contractions in Hilbert space. Nonlinear Anal. 67, 2258-2271 (2007)

5. Cho, YJ, Kang, SM, Qin, X: Some results on k-strictly pseudo-contractive mappings in Hilbert spaces. Nonlinear Anal. 70, 1956-1964 (2009)

6. Jung, JS: Strong convergence of iterative methods for $k$-strictly pseudo-contractive mappings in Hilbert spaces. Appl. Math. Comput. 215, 3746-3753 (2010)

7. Jung, JS: Some results on a general iterative method for $k$-strictly pseudo-contractive mappings. Fixed Point Theory Appl. 2011, 24 (2011). doi:10.1186/1687-1812-2011-24

8. Jung, JS: A general iterative method with some control conditions for $k$-strictly pseudo-contractive mappings. J. Comput. Anal. Appl. 14, 1165-1177 (2012)

9. Morales, $\mathrm{CH}$, Jung, JS: Convergence of paths for pseudo-contractive mappings in Banach spaces. Proc. Am. Math. Soc. 128, 4311-4319 (2000)

10. Deutch, F, Yamada, I: Minimizing certain convex functions over the intersection of the fixed point sets of nonexpansive mappings. Numer. Funct. Anal. Optim. 19, 33-56 (1998)

11. Xu, HK: An iterative approach to quadratic optimization. J. Optim. Theory Appl. 116, 659-678 (2003) 
12. Yamada, I: The hybrid steepest descent for the variational inequality problems over the intersection of fixed points sets of nonexpansive mappings. In: Butnariu, D, Censor, Y, Reich, S (eds.) Inherently Parallel Algorithms in Feasibility and Optimization and Their Applications, pp. 473-504. Elsevier, New York (2001)

13. Marino, G, Xu, HK: A general iterative method for nonexpansive mappings in Hilbert spaces. J. Math. Anal. Appl. 318, 43-56 (2006)

14. Moudafi, A: Viscosity approximation methods for fixed-points problem. J. Math. Anal. Appl. 241, 46-55 (2000)

15. Tian, M: A general iterative algorithm for nonexpansive mappings in Hilbert spaces. Nonlinear Anal. 73, 689-694 (2010)

16. Tian, M: A general iterative method based on the hybrid steepest descent scheme for nonexpansive mappings in Hilbert spaces. In: 2010 International Conference on Computational Intelligence and Software Engineering (CiSE 2010), 5677064 (2010)

17. Ceng, LC, Ansari, QH, Yao, JC: Some iterative methods for finding fixed points and for solving constrained convex minimization problems. Nonlinear Anal. 74, 5286-5302 (2011)

18. Ceng, LC, Guu, SM, Yao, JC: A general composite iterative algorithm for nonexpansive mappings in Hilbert spaces. Comput. Math. Appl. 61, 2447-2455 (2011)

19. Shioji, N, Takahashi, W: Strong convergence of approximated sequences for nonexpansive mappings in Banach spaces. Proc. Am. Math. Soc. 125(12), 3641-3645 (1997)

20. Liu, LS: Iterative processes with errors for nonlinear strongly accretive mappings in Banach spaces. J. Math. Anal. Appl. 194, 114-125 (1995)

21. Xu, HK: Iterative algorithms for nonlinear operators. J. Lond. Math. Soc. 66, 240-256 (2002)

22. Goebel, K, Kirk, WA: Topics in Metric Fixed Point Theory. Cambridge Stud. Adv. Math., vol. 28. Cambridge Univ. Press, Cambridge (1990)

23. Zhou, $\mathrm{H}$ : Convergence theorems of fixed points for $k$-strict pseudo-contractions in Hilbert spaces. Nonlinear Anal. 69 456-462 (2008)

24. Cho, YJ, Kang, SM, Zhou, HY: Some control conditions on iterative methods. Commun. Appl. Nonlinear Anal. 12(2), 27-34 (2005)

25. Jung, JS, Cho, YJ, Agarwal, RP: Iterative schemes with some control conditions for a family of finite nonexpansive mappings in Banach space. Fixed Point Theory Appl. 2005(2), 125-135 (2005)

26. Jitpeera, T, Kumam, P: The shrinking projection method for a system of generalized mixed equilibrium problems and fixed point problems for pseudocontractive mappings. J. Inequal. Appl. 2011, 840319 (2011)

27. Jitpeera, T, Kumam, P: A composite iterative method for generalized mixed equilibrium problems and variational inequality problems. J. Comput. Anal. Appl. 13(2), 345-361 (2011)

28. Onjai-Uea, N, Jaiboon, C, Kumam, P: A relaxed hybrid steepest descent method for common solutions of generalized mixed equilibrium problems and fixed point problems. Fixed Point Theory Appl. 2011, 32 (2011)

29. Onjai-Uea, N, Jaiboon, C, Kumam, P, Humphries, UW: Convergence of iterative sequences for fixed points of an infinite family of nonexpansive mappings based on a hybrid steepest descent methods. J. Inequal. Appl. 2012,101 (2012)

30. Onjai-Uea, N, Kumam, P: Convergence theorems for generalized mixed equilibrium and variational inclusion problems of strict-pseudocontractive mappings. Bull. Malays. Math. Sci. Soc. (2) 36(4), 1049-1070 (2013)

31. Witthayarat, U, Jitpeera, T, Kumam, P: A new modified hybrid steepest-descent method by using a viscosity approximation method with a weakly contractive mapping for a system of equilibrium problems and fixed point problems with minimization problems. Abstr. Appl. Anal. 2012, 206345 (2012)

32. Witthayarat, U, Kim, JK, Kumam, P: A viscosity hybrid steepest-descent method for a system of equilibrium problems and fixed point for an infinite family of strictly pseudo-contractive mappings. J. Inequal. Appl. 2012, 224 (2012)

10.1186/1687-1812-2014-173

Cite this article as: Jung: A general composite iterative method for strictly pseudocontractive mappings in Hilbert spaces. Fixed Point Theory and Applications 2014, 2014:173

\section{Submit your manuscript to a SpringerOpen ${ }^{\ominus}$ journal and benefit from:}

- Convenient online submission

- Rigorous peer review

- Immediate publication on acceptance

Open access: articles freely available online

- High visibility within the field

- Retaining the copyright to your article 